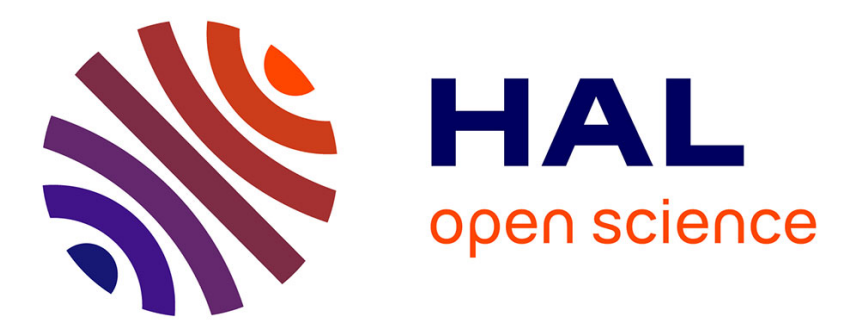

\title{
Collapse dynamics and runout of dense granular materials in a fluid
}

Vincent Topin, Yann Monerie, Frédéric Perales, Farhang Radjai

\section{To cite this version:}

Vincent Topin, Yann Monerie, Frédéric Perales, Farhang Radjai. Collapse dynamics and runout of dense granular materials in a fluid. Physical Review Letters, 2012, 109, pp.188001. 10.1103/PhysRevLett.109.188001 . hal-00689730

\section{HAL Id: hal-00689730 https://hal.science/hal-00689730}

Submitted on 20 Apr 2012

HAL is a multi-disciplinary open access archive for the deposit and dissemination of scientific research documents, whether they are published or not. The documents may come from teaching and research institutions in France or abroad, or from public or private research centers.
L'archive ouverte pluridisciplinaire HAL, est destinée au dépôt et à la diffusion de documents scientifiques de niveau recherche, publiés ou non, émanant des établissements d'enseignement et de recherche français ou étrangers, des laboratoires publics ou privés. 


\title{
Collapse dynamics and runout of dense granular materials in a fluid
}

\author{
V. Topin ${ }^{a, b}$, Y. Monerie ${ }^{a, b}$, F. Perales ${ }^{a, b}$, F. Radjaï ${ }^{b, c}$ \\ ${ }^{a}$ Institut de Radioprotection et Sûreté Nucléaire (IRSN), PSN, \\ CE Cadarache, BP3 - 13115 St Paul-Lez-Durance Cedex, France \\ ${ }^{b}$ Laboratoire de Micromécanique et Intégrité des STructures (MIST), \\ CNRS-IRSN-Université Montpellier 2, France and \\ ${ }^{c}$ Laboratoire de Mécanique et Génie Civil, CNRS-Université Montpellier 2, \\ Place Eugène Bataillon, 34095 Montpellier cedex 5, France.
}

(Dated: April 16, 2012)

\begin{abstract}
We investigate the effect of an ambient fluid on the dynamics of collapse and spread of a granular column simulated by means of the contact dynamics method interfaced with computational fluid dynamics. The runout distance is found to increase as a power law with the aspect ratio of the column and, surprisingly, for a given aspect ratio and packing fraction, it may be similar in the grain-inertial and fluid-inertial regimes but with considerably longer duration in the latter case. We show that the effect of fluid in viscous and fluid-inertial regimes is to both reduce the kinetic energy during collapse and enhance the flow by lubrication during spread. Hence, the runout distance in a fluid may be below or equal to that in the absence of fluid due to compensation between those effects.
\end{abstract}

Modeling the dynamics of dense granular materials submerged in a fluid represents a major challenge both in the context of geological flows and for a better control of wet processing in powder technology. Most natural destructive events involve the destabilization and flow of dense and polydisperse granular materials (sand, gravels or rocks) saturated by or immersed in water (slurries, submarine avalanches, debris flow) [1, 2]. The prediction of the runout distance of such hydro-granular flows according to their initial composition and geometry is crucial for risk assessment. Likewise, very basic processes in food and pharmaceutical industries rely on the transport of a powder or a collection of aggregates in a liquid which plays the role of lubricant or binder. The grainscale mechanisms of transport and segregation in such processes are generally poorly understood [3]. The dispersion of fuel fragments in the coolant water during a hypothetic nuclear accident is another example of the intricate grain/fluid mixing process in extreme conditions, which remains a real unknown for the design of modern pressurized water reactors.

The presence of a fluid phase in a granular medium has profound effects on its mechanical behavior. In dry granular media the rheology is governed by grain inertia and static stresses sustained by the contact network depending on the shear-rate and confining pressure, respectively [4]. As the fluid inertia and viscosity come into play, complications arise as a result of contradictory effects. On one hand, the fluid may delay the onset of granular flow or prevent the dispersion of the grains by developing negative pore pressures $[1,[5,[6]$. On the other hand, the fluid lubricates the contacts between grains, enhancing in this way the granular flow, and it has a retarding effect at the same time by inducing drag forces on the grains [7].

In this Letter, we rely on extensive numerical simulations to analyze the relative importance of grain inertia, fluid inertia and viscous effects in a dynamic test consisting of a granular column allowed to collapse and spread on a horizontal plane under its own weight. This choice was motivated by well-documented experimental and numerical data for similar tests but with dry granular materials $[8-11]$. A common observation is that the terminal morphology of deposits is independent of grain size and packing fraction, and the runout distance grows in a nontrivial manner with the initial aspect ratio. Our simulations reveal a nearly similar behavior in the presence of an ambient fluid but with a rather complex dependence on the fluid inertia and viscosity.

The simulations were performed by means of a recently developed model coupling the contact dynamics method 12 14 for discrete-element modeling of the grains and their interactions with the finite-element method for the integration of Navier-Stokes equations in 2D [6]. The coupling is based on the "fictitious domain" approach in which the fluid domain is extended to that of grains, and the rigid-body motion of the grains is imposed by means of distributed Lagrange multipliers [15, 16]. Sample movies of the simulations analyzed in this Letter are available at www.cgp-gateway.org/ref013. Note that, due to a broad fluid domain with frictional contact interactions, these simulations are cpu-intensive and take several days with a parallelized version of the software running on several tens of processors.

The granular samples are composed of disks of mean diameter $d=10^{-3} \mathrm{~m}$ with a weak size polydispersity $\Delta d / d=0.8$. The disks are assembled in a rectangular domain of width $R_{0}$ and height $H_{0}$. The fluid domain is rectangular with dimensions varying between $150 d \times 60 d$ and $300 d \times 150 d$. The grains are assumed to be perfectly rigid with normal and tangential restitution coefficients set to zero. The Coulomb friction coefficient is fixed to 0.3 between grains and with the walls. The fluid density $\rho_{f}$ is that of water $\rho_{H_{2} O}$ and we set the density 


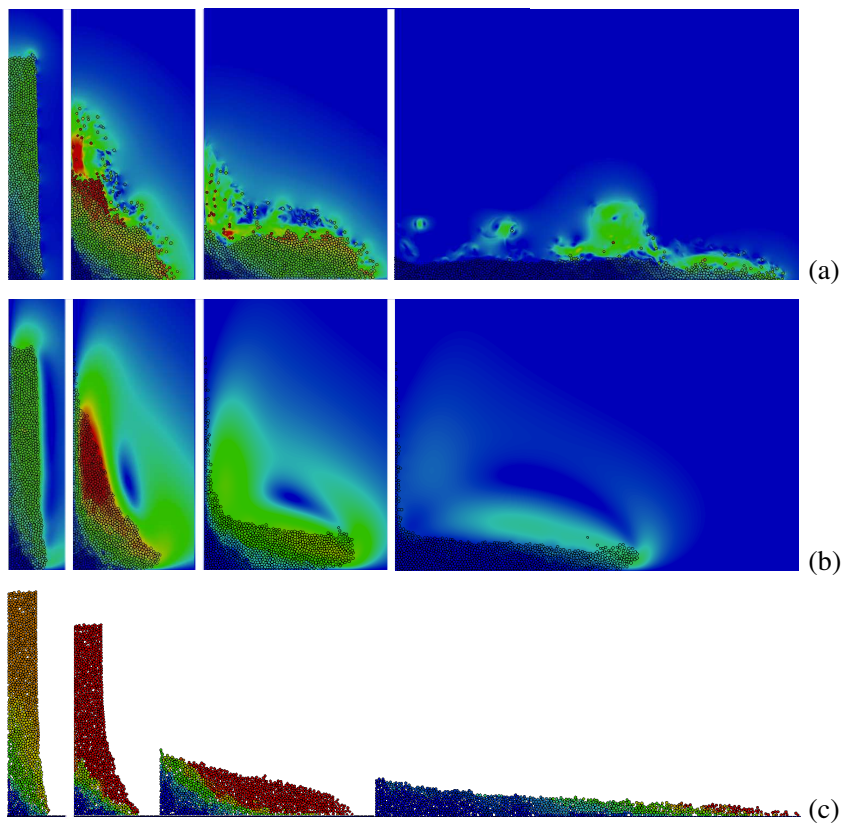

FIG. 1. (Color online) Successive instants of the collapse of a column of aspect ratio $a=8$ in (a) fluid-inertial, (b) viscous and (c) grain-inertial regimes. The grains and fluid are colored according to the amplitude of their velocities.

of grains $\rho_{s}=2.6 \rho_{f}$, roughly corresponding to rock debris in water. For each aspect ratio $a=H_{0} / R_{0}$, three simulations were performed: one without fluid and two with fluid for two values of the viscosity $\eta=\eta_{\mathrm{H}_{2} \mathrm{O}}$ and $10^{3} \eta_{H_{2} \mathrm{O}}$. These simulations correspond to grain-inertial, fluid-inertial and viscous regimes, respectively [17, 18]. The width of the column is fixed to $R_{0}=11.5 d$ and $a$ varies in the range $] 0 ; 10]$. The largest number of grains is 1360 for $a=10$. Since we focus in this paper on the influence of the column aspect ratio and grain/fluid regimes on the runout, the packing fraction is set to $\phi=0.8$ in all simulations. Note that only $d$, the grain mass $m$ and the gravity $g$ keep the same values in all regimes, and for this reason we normalize the lengths by $d$, the times by $\sqrt{d / g}$, the velocities by $\sqrt{g d}$, the energies by $m g d$ and the viscosities by $m \sqrt{g / d}$.

Figure 1 displays successive snapshots of the collapse and flow of grains for $a=8$ in the three grain/fluid regimes. The grains collapse vertically and jam in a heap that spreads along the plane and finally stops. Convective rolls are induced in fluid by granular flow. The three phases (collapse, heap and spread) are clearly evidenced in Fig. 2 where grain trajectories are shown together with the mean kinetic energy per grain $E_{c x}=\left\langle m v_{x}^{2} / 2\right\rangle$ and $E_{c y}=\left\langle m v_{y}^{2} / 2\right\rangle$ carried by the horizontal and vertical grain velocity components, respectively, as a function of time. The vertical collapse is characterized by the fast growth of $E_{c y}$ and negligible $E_{c x}$. The latter begins to increase only at the peak value of $E_{c y}$, and in a short time

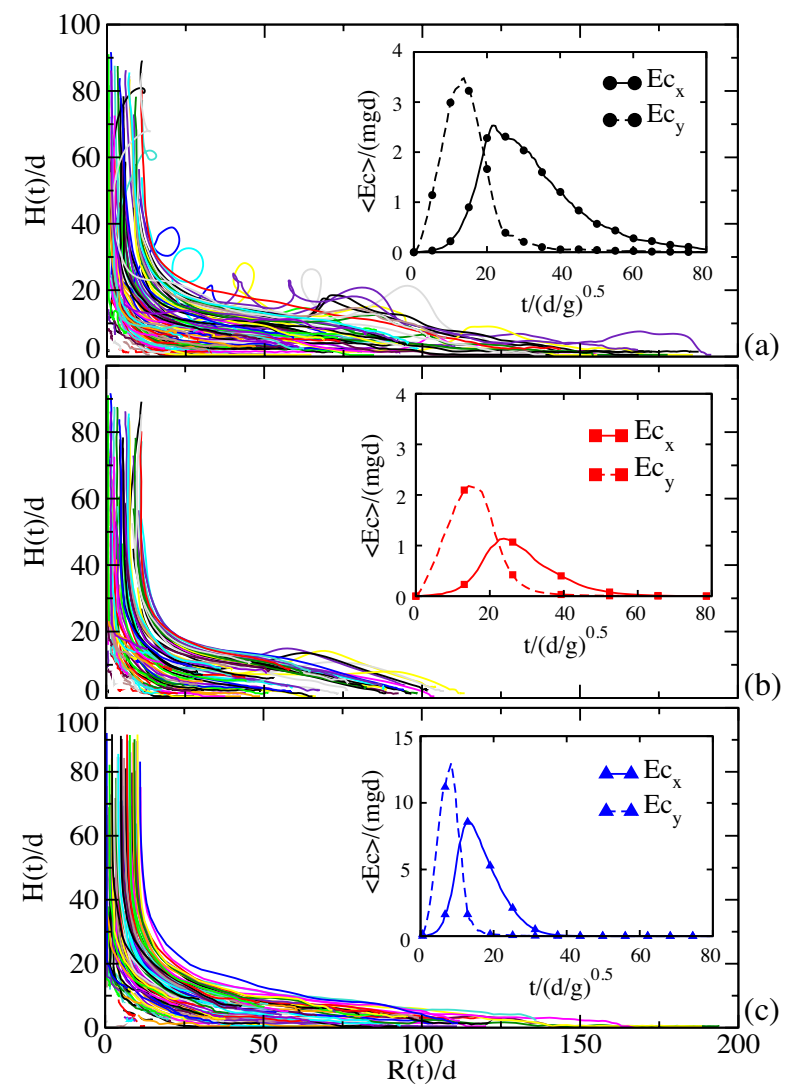

FIG. 2. Grain trajectories (main plots) in the fluid-inertial (a), viscous (b) and grain-inertial (c) regimes for $a=8$. Only $10 \%$ of trajectories are plotted. The insets show the evolution of the mean kinetic energy per grain carried by the horizontal $(x)$ and vertical $(y)$ components of grain velocities.

interval extending from this point to the peak value of $E_{c x}$, most of the kinetic energy is transformed from vertical direction to horizontal direction. This short interval defines unambiguously the heap phase. Finally, the spread phase is reflected in the long tail of $E_{c x}$ falling off from its maximum to zero. For small aspect ratios $(a<4)$, we observe no distinct collapse phase.

The evolution of $E_{c y}$ indicates that the grains do not reach their Stokes velocity in the fluid. The mean vertical velocity of the ten initially highest grains is plotted as a function of time for $a=8$ in Fig. 3. We note that, regardless of the grain/fluid regime, the collapse obeys a power law $\left\langle V_{y}\right\rangle_{10} \propto t^{\beta}$ over nearly one decade with $\beta \simeq 1$ in the grain-inertial regime, corresponding to a ballistic fall, $\beta \simeq 0.95$ in the fluid-inertial regime and $\beta \simeq 0.75$ in the viscous regime. This is an indication of the collective collapse of the grains with liquid as binding agent.

Figure 4 shows the total normalized runout distance $\left(R_{\text {stop }}-R_{0}\right) / R_{0}$ and runout duration $t_{\text {stop }}$ as a function of $a$. The behavior is similar in all regimes: for small aspect ratios $(a<4)$ the runout distance increases linearly: $\left(R_{\text {stop }}-R_{0}\right) / R_{0}=\lambda_{1} a$ with $\lambda_{1} \simeq 2.45$ for the 


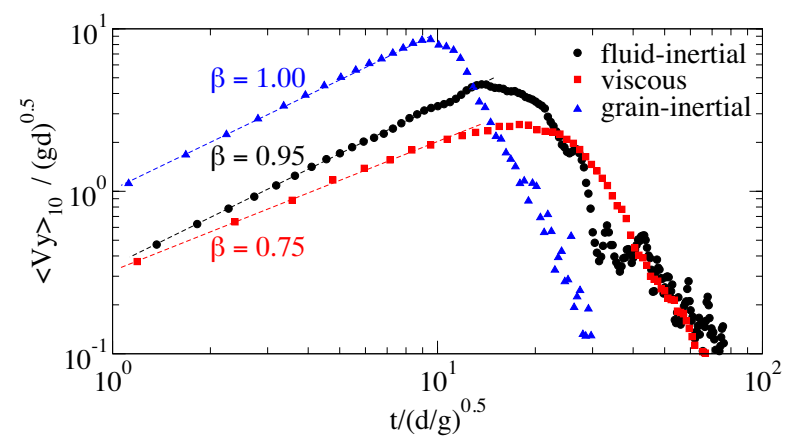

FIG. 3. Average vertical velocity of the ten initially highest grains as a function of time for $a=8$ in different grain/fluid regimes.

grain-inertial and fluid-inertial regimes and $\simeq 1.21$ in the viscous regime. For larger aspect ratios, the runout distance follows a power law $R_{\text {stop }} \propto \lambda_{2} a^{\alpha}$ with $\alpha \simeq 0.6 \pm 0.1$ and $\lambda_{2} \simeq 4.3$ in the the grain-inertial and fluid-inertial regimes and $\alpha \simeq 0.87 \pm 0.1$ and $\lambda_{2} \simeq 1.5$ in the viscous regime. It is remarkable that the values of $\lambda_{1}, \lambda_{2}$ and $\alpha$ in the grain-inertial and fluid-inertial regimes are identical to those reported in the dry case for narrow or $2 \mathrm{D}$ flows 8 -11]. The equality of the runout distance between grain-inertial and fluid-inertial regimes contradicts at first sight the fact that underwater avalanches have a longer runout distance [2]. The change of behavior between small and large aspect ratios also appears in $t_{\text {stop }}$, which seems to increase linearly but with two different slopes as a function of $a$. However, unlike the runout distance, the duration is significantly shorter in the grain-inertial regime than in the fluid-inertial regime for all values of $a$. Note also that, unexpectedly, the runout duration is shorter in the viscous regime than in the fluid-inertial regime.

The spatiotemporal evolution of the grains and their kinetic energy, evidenced in Fig. 22 suggests that the runout may be portrayed as resulting from the transformation of (part of) the initial potential energy into the peak kinetic energy $E_{c x}^{\max }$ that controls in turn the subsequent runout along the plane. The process can thus be split by analyzing separately the dependence of $E_{c x}^{\max }$ with respect to $a$, on one hand, and $R_{\text {stop }}$ as a function of $E_{c x}^{\max }$, on the other hand. These functions are plotted from all simulation data in Fig. [5. We see that, irrespective of the grain/fluid regime, $E_{c x}^{\max }$ is a growing function of $a$ with a transition around $a \simeq 4$. This is consistent with the fact that the grains do not reach their Stokes velocity in the fluid (Fig. 3) since otherwise the kinetic energy per grain would not depend on $a$ unless probably at low $a . E_{c x}^{\max }$ is considerably higher in the grain-inertial regime, indicating that part of the potential energy in the presence of the fluid is dissipated during vertical collapse due to viscous friction and contact interactions.

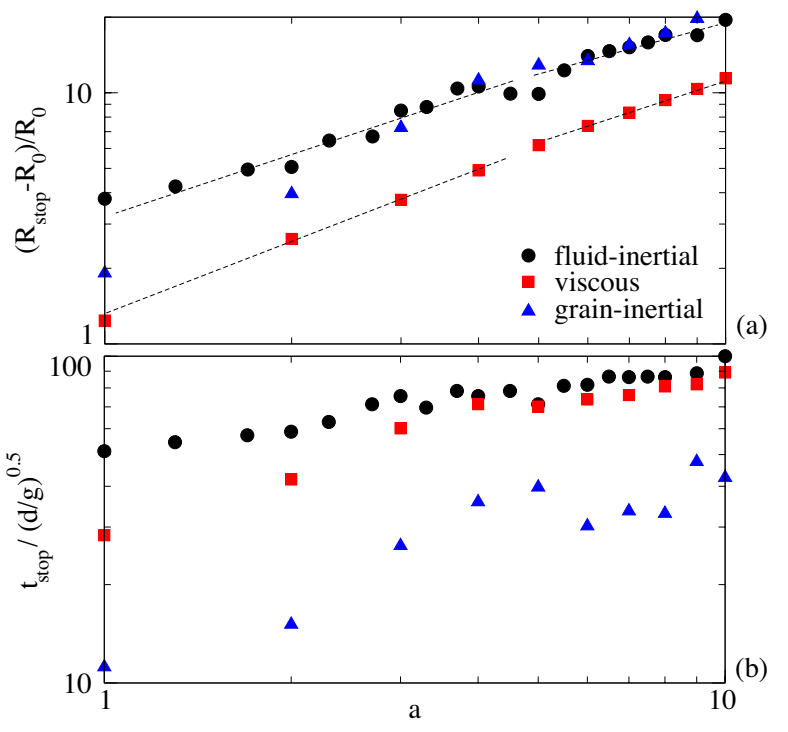

FIG. 4. Normalized runout distance (a) and duration (b) as a function of the aspect ratio in different grain/fluid regimes.

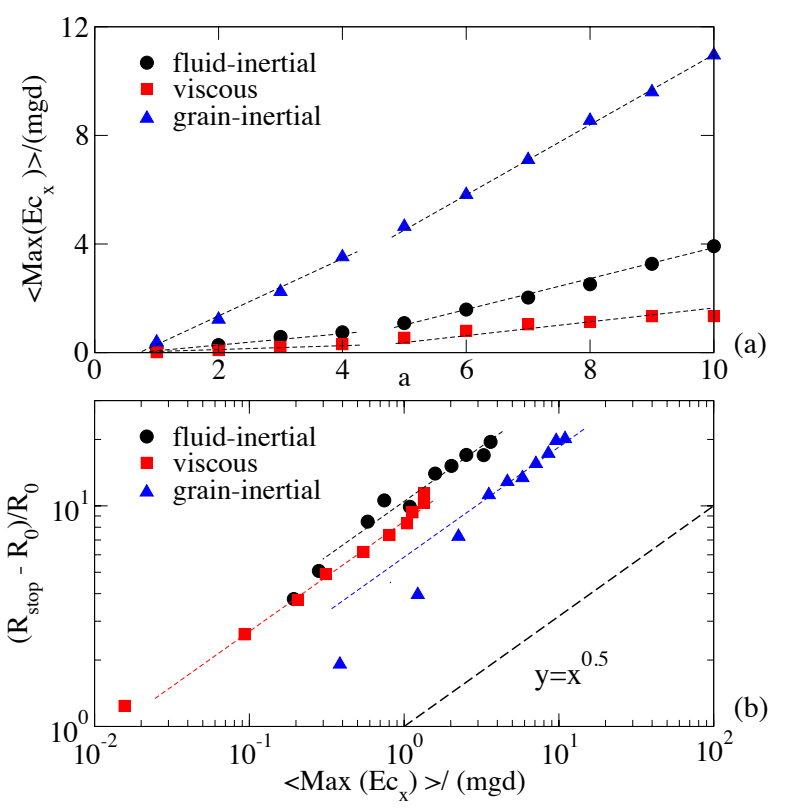

FIG. 5. (a) Peak value of the mean horizontal kinetic energy per grain in different grain/fluid regimes as a function of aspect ratio. (b) Normalized runout distance as a function of the peak value of the horizontal kinetic energy per grain.

Figure 5(b) reveals a very simple dependence of the runout distance with respect to the maximum kinetic energy at large aspect ratios. In all regimes, in exception to low energies in the grain-inertial regime, the runout distance increases as a power law $R_{\text {stop }} \propto\left(E_{c x}^{\max }\right)^{\gamma}$ with $\gamma=0.50 \pm 0.05$. When the runout distance is compared among the three regimes not for the same initial aspect 
ratio as in Fig. 4 but rather at the same level of $E_{c x}^{\max }$, it has its lowest value in the grain-inertial regime, largest value in the fluid-inertial regime and intermediate values in the viscous regime. This result is physically plausible as the fluid has a lubricating effect at the contacts and fluidizes the grain/fluid mixture. These effects are more pronounced in the fluid-inertial regime and, what is more, the viscosity is lower. For this reason, $R_{\text {stop }}$ is larger in the fluid-inertial regime than in the viscous regime.

The unexpected traits observed in Fig. 4 can now be understood in the light of the collapse and runout data of Fig. 5. For a given aspect ratio, the grains acquire the highest kinetic energy in the grain-inertial regime due to the lack of fluid dissipation during vertical collapse. This extra kinetic energy is high enough to propel the heap, in spite of a high frictional dissipation, over a distance that can be longer than the runout distance in the fluidinertial regime. In the latter case, the grains begin to spread with a lower kinetic energy but dissipate much less energy due to contact lubrication. In the viscous regime, for the same aspect ratio, the kinetic energy available for spreading is still lower and the dissipation due to viscous drag is higher, leading thus to a much shorter runout distance.

The scaling of $R_{\text {stop }}$ with $E_{c x}^{\max }$ at large aspect ratios suggests that, in a mean-field picture of the spread phase, each grain is on average subject to a viscous drag force $f_{x}=-k v_{x}$ (here in $2 \mathrm{D}$ ), where $k$ is the effective viscosity of the mixture. Indeed, solving the equation of motion $m \dot{v}_{x}=f_{x}$ for a single grain (for example, the grain at the tip of the slurry) with initial velocity $v_{\max }=\sqrt{2 E_{c x}^{\max } / m}$ and assuming that $R_{0}$ is the position of the grain when the $E_{c x}$ takes its peak value, yields $R_{\text {stop }}-R_{0}=\sqrt{2 m E_{c x}^{\max }} / k$. The values of $k$ may be estimated from the data for each regime. We get $k_{f i} \simeq 0.012$ for the fluid-inertial regime, $k_{v} \simeq 0.014$ for the viscous regime and $k_{g i} \simeq 0.018$ for the grain-inertial regime in dimensionless units. $k_{g i}$ may be attributed to collisions between grains at high shear rates [4]. The value of $k_{f i}$ is larger than $\eta_{H_{2} O} \simeq 0.0018$ as expected for a dense suspension of grains. But $k_{v}$ is well below the fluid viscosity $10^{3} \eta_{H_{2} O}$ in the viscous regime. This picture based on the dynamics of a single grain can be improved by adjusting the mass in order to account for the collective flow of the grains. However, a depth-averaged model of the transient dynamics of the slurry, as that developed in [9] for dry grains, should provide a more systematic approach for a better understanding of the data.

In summary, in this Letter we used the dam-break configuration as a benchmark test to investigate for the first time by coupled numerical simulations in $2 \mathrm{D}$ the influence of an ambient fluid on transient granular dynamics. The effect of fluid in both viscous and fluid-inertial regimes is to reduce the kinetic energy during collapse and to enhance the flow by lubrication during spread. Hence, the runout distance in a fluid for a given geome- try of the column may be below or equal to that in the absence of fluid due to compensation between those effects. This shows that the trigger mechanism plays a crucial role in the runout distance in the presence of a fluid as previously pointed out also in the dry case [10]. In our dam-break geometry, the avalanche is triggered by the energy released as a result of the spontaneous collapse of the column. The generic effect of fluid on the runout might more easily be investigated by assuming an arbitrary source of kinetic energy (earthquake, tsunami,...) in a granular assembly initially at rest. The simulations provide a convenient tool that can be exploited to investigate in such generic configurations the influence of grain/fluid characteristics such as particle size distribution.

We gratefully acknowledge A. Wachs (IFP, Lyon, France) for his essential help in numerical developments and J.-Y. Delenne (LMGC, Montpellier, France) for helpful discussions.

[1] M. Iverson, M. E. Reid, N. R. Iverson, R. G. LaHusen, M. Logan, J. E. Mann, and D. L. Brien, science 290 (2000).

[2] F. Legros, Engineering Geology 63, 301 (2002).

[3] M. J. Rhodes, Introduction to particle technology (John Wiley \& Sons, Chichester, 1998).

[4] G.D.R Midi, European Physical Journal E 14, 341 (2004).

[5] M. Pailha, M. Nicolas, and O. Pouliquen, Physics of fluids 20 (2008).

[6] V. Topin, F. Dubois, Y. Monerie, F. Perales, and A. Wachs, Journal of Non-Newtonian Fluid Mechanics 166, 63 (2011).

[7] B. Maury and A. Lefebvre-Lepot, "Discrete-element modeling of granular materials" (Iste-Wiley London, 2011) Chap. Close interaction of immersed grains, pp. 329-345.

[8] E. Lajeunesse, J. B. Monnier, and G. M. Homsy, Physics of fluids 17, 2371 (2005).

[9] N. J. Balmforth and R. R. Kerswell, J. Fluid Mech. 538, 399 (2005).

[10] L. Staron and E. J. Hinch, Granular Matter , 205 (2007).

[11] L. Lacaze and R. Kerswell, Physical Review Letter 102, 108305 (2009).

[12] J. J. Moreau, European Journal of Mechanics A/Solids 4, 93 (1994).

[13] M. Jean, V. Acary, and Y. Monerie, Philosophical Transactions of Royal Society London A 359, 2497 (2001).

[14] F. Radjai and V. Richefeu, Mechanics of Materials 41, 715 (2009).

[15] R. Glowinski, T. W. Pan, T. I. Hesla, and D. D. Joseph, International Journal of Multiphase Flow 25, 755 (1999)

[16] A. Wachs, Journal of Engineering Mathematics 38-1, 131 (2011).

[17] S. Courrech du Pont, P. Gondret, G. Perrin, and M. Rabaud, Physical Review Letter 90, 044301 (2003).

[18] C. Cassar, M. Nicolas, and O. Pouliquen, Physics of fluids 17, 103301 (2005). 